\title{
Gaseous Haloes: Linking Galaxies to the IGM
}

\author{
Filippo Fraternali ${ }^{\text {a,d }}$ James Binney ${ }^{\mathrm{b}}$ Tom Oosterloo $^{\text {c,e }}$ \\ Renzo Sancisi d,e \\ ${ }^{a}$ Department of Astronomy, University of Bologna, via Ranzani 1, 40127, Bologna, \\ Italy \\ ${ }^{\mathrm{b}}$ Rudolf Peierls Centre for Theoretical Physics, 1 Keble Rd, Oxford, OX1 3NP, \\ $U K$ \\ cASTRON, Postbus 2, 7990 AA Dwingeloo, NL \\ ${ }^{\mathrm{d}}$ INAF - Astronomical Observatory, via Ranzani 1, 40127, Bologna, Italy \\ ${ }^{\mathrm{e}}$ Kapteyn Astronomical Institute, Postbus 800, 9700 AV Groningen, NL
}

\begin{abstract}
In recent years evidence has accumulated that nearby spiral galaxies are surrounded by massive haloes of neutral and ionised gas. These gaseous haloes rotate more slowly than the disks and show inflow motions. They are clearly analogous to the High Velocity Clouds of the Milky Way. We show that these haloes cannot be produced by a galactic fountain process (supernova outflows from the disk) where the fountain gas conserves its angular momentum. Making this gas interact with a pre-existing hot corona does not solve the problem. These results point at the need for a substantial accretion of low angular momentum material from the IGM.
\end{abstract}

Key words: spiral galaxies, haloes, NGC891, gas dynamics

\section{Introduction}

Recent deep neutral hydrogen (H I) observations of several nearby spiral galaxies (e.g. NGC 891, Swaters. Sancisi. \& van der Hulst (1997); NGC 2403, Fraternali et al. (2002); M31, Westmeier, Braun, \& Thilker (2005); UGC 7321, Matthews \& Wood (2003)) indicate that a large fraction of the neutral gas is located outside the plane of the disk. Such "halo" gas has peculiar kinematics: it rotates more slowly than the gas in the disk (Swaters, Sancisi, \& van der Hulst, 1997) and

Email address: filippo.fraternali@unibo.it (Filippo Fraternali). 
shows strong vertical motions from and/or towards the disk (Boomsma et al., 2005). In two galaxies (NGC 2403, Fraternali et al. (2001); and NGC 4559, Barbieri et al. (2005)) the halo gas also shows an overall radial inflow towards the centre of the galaxy. Halo gas is also detected in optical emission lines (e.g. Hoopes. Walterbos \& Rand, 1999; Rossa \& Dettmar, 2003) with kinematics similar to that of the neutral gas (Heald et al., 2006b) and in X-rays (e.g. Strickland et al., 2004). Halo gas around external galaxies has the same properties (masses and velocities) of the Intermediate and High Velocity Clouds (IVCs and HVCs) of the Milky Way (Wakker \& van Woerden, 1997).

What is the origin of these extended gaseous haloes? In galaxies like NGC 891 gas is ejected from the disk by stellar activity producing a circulation called galactic fountain (Shapiro \& Field, 1976; Bregman, 1980). However, it is now clear that a "pure" (ballistic) galactic fountain cannot explain the kinematics of the halo gas (Fraternali \& Binney, 2006; Heald et al., 2006b). In particular there is a need for the fountain gas to loose part of its angular momentum. Such a loss can be achieved in two ways: 1) interaction between the fountain clouds and a pre-existing hot corona or 2) interaction with accreting material. We explore here the first possibility.

\section{The Structure of the HI halo of NGC891}

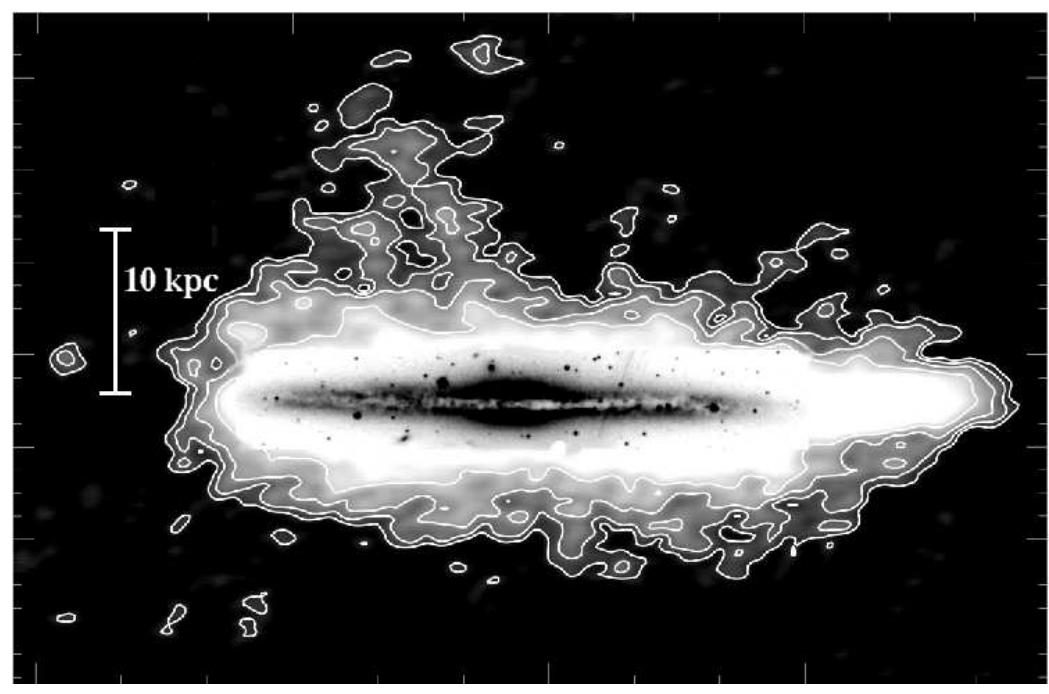

Fig. 1. Optical DSS image (grey-scale) and total H I map (contours+negative grey-scale) of NGC 891 . H I contours are: $1,2,4,8,16 \times 10^{19}$ atoms $\mathrm{cm}^{-2}$. The beam size is $25^{\prime \prime}=1.15 \mathrm{kpc}$.

New deep H I observations of the nearby edge-on spiral galaxy NGC 891 obtained with the Westerbork Synthesis Radio Telescope show an extended and massive (25\% of the total H I mass) halo component of neutral gas (Oosterloo, Fraternali \& Sancisi, 2007). 
Fig. 1 shows the total H I map of NGC 891 (countours + white shade) with overlaid an optical image (grey-scale). The map is rotated 67 degrees counterclock wise with respect to the on-sky orientation. The neutral gas everywhere extends to large heights $(z \sim 8 \mathrm{kpc})$ from the plane. Some features, in particular a long filament, extend to much larger distances $(\sim 20 \mathrm{kpc}$ from the plane). The kinematics of the halo gas in NGC 891 is characterized by a regular decrease in rotational velocity with $z$ (gradient of $\sim 15 \mathrm{~km} \mathrm{~s}^{-1} \mathrm{kpc}^{-1}$ ) (Fraternali et al., 2005). Most of the high latitude features, i.e. the filament, are at roughly the systemic velocity.

\section{Modelling the halo gas}

Fraternali \& Binney (2006) have presented a model for the formation of the halo gas in spiral galaxies. The model is built by integrating the orbits of gas clouds that are ejected from the disk of a spiral galaxy, and then move ballistically up into the halo and back down to the disk. At each timestep, the positions and velocities of the particles are projected along the line of sight to produce an artificial cube, that can be compared with observed H I data cubes.

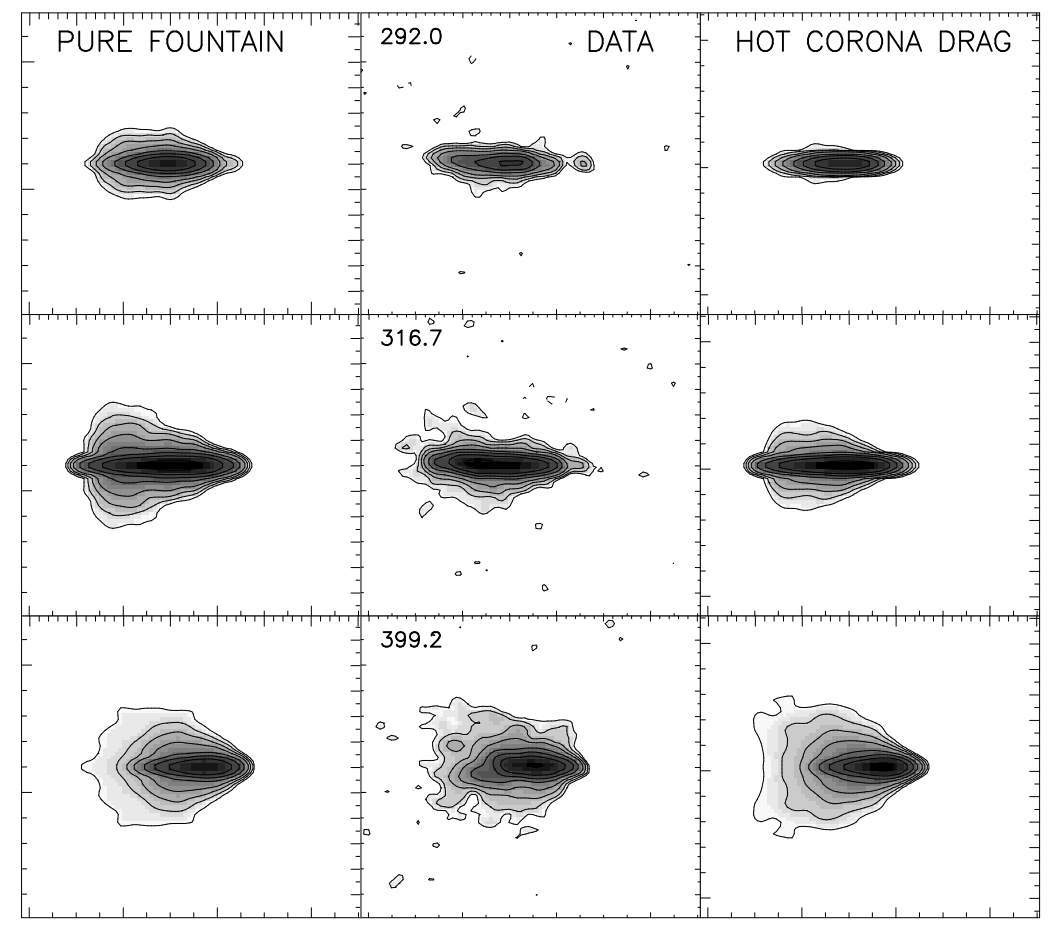

Fig. 2. Comparison between three representative channel maps for NGC 891 (central column) and two models: pure galactic fountain (left column) and galactic fountain interacting with the hot corona (right column). Heliocentric velocities $(\mathrm{km} / \mathrm{s})$ are on the top left corners of the data. 


\subsection{Pure galactic fountain}

At first we consider a model with only gravitational effects (ballistic galactic fountain) (Fraternali \& Binney, 2006). Fig. 2 shows three channel maps of NGC 891 (central column) compared with the prediction of such a fountain model (left column). The top row shows a channel map at velocity far away from the systemic velocity $\left(\mathrm{v}_{\mathrm{sys}}=530 \mathrm{~km} \mathrm{~s}^{-1}\right)$. Here the gas is rotating at high speed and it is all concentrated in the disk. The more we move towards the systemic velocity the more the maps become thicker; in other words the halo gas rotates much more slowly than the disk gas. Clearly the maps produced by the pure fountain model show more fast-rotating halo gas than the data.

\subsection{Interaction with the hot corona}

We consider the interaction (drag) between the fountain gas and a pre-existing hot corona rotating more slowly than the disk (Fraternali \& Binney, in preparation). We model the corona as a plasma at $T=2.7 \times 10^{6} \mathrm{~K}$ in equilibrium in the axisymmetric potential. The plasma density is normalized using the observed X-ray luminosity of NGC 891 (Strickland et al., 2004). Fig. 2 (third column) shows the result of such a model with a hot corona rotating a constant speed $\mathrm{v}_{\phi}(R)=100 \mathrm{~km} \mathrm{~s}^{-1}$. Clearly this produces an improvement with respect to the pure fountain model.

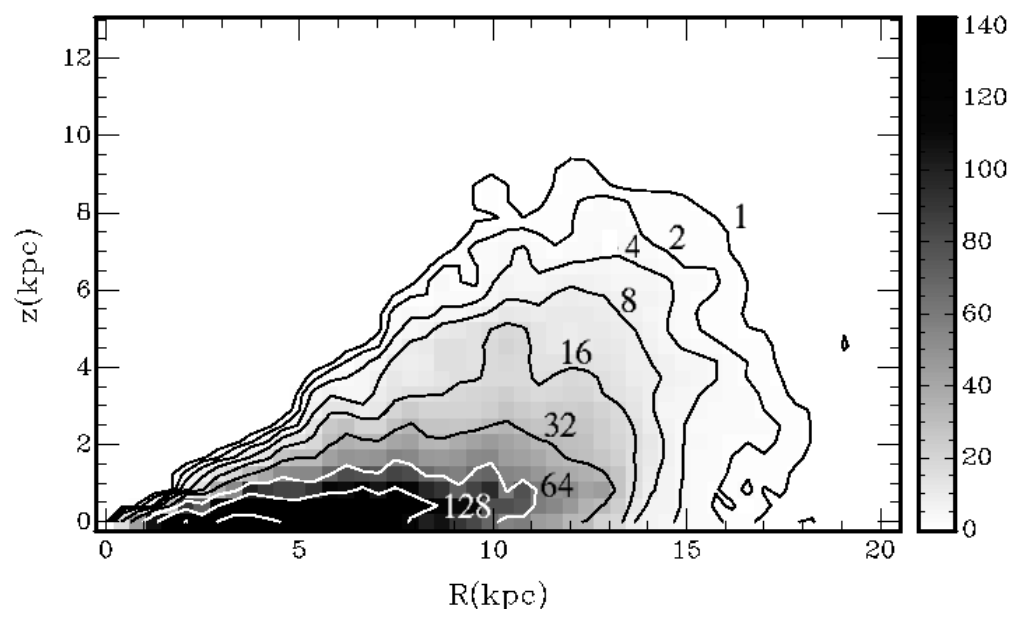

Fig. 3. Azimuthal velocities acquired by the hot corona per Myr due to the transferring of angular momentum from the fountain gas.

The key point of the above model is that the fountain particles transfer part of their angular momentum to the hot corona. However, can the corona absorb such momentum? Fig. 3 shows the azimuthal velocity acquired by the inner corona after a Myr as a function of the coordinates $R$ and $z$. The spin-up time of the inner halo is very rapid; within a time $t_{\text {spinup }} \lesssim 1 \mathrm{Myr}$ the inner 
corona co-rotates with the disk and the drag vanishes. Therefore the hot corona cannot be responsible for the observed lag of the halo gas.

\section{Conclusions}

We have seen that: 1) the spiral galaxy NGC891 is surrounded by a massive halo of neutral gas extending up to more than $8 \mathrm{kpc}$ from the plane; 2) extraplanar gas such as that in NGC 891 seems to be common in spiral galaxies and is analogous to the HVCs of the Milky Way; 3) models purely based on a galactic fountain, with or without interaction with the hot corona, fail to reproduce the data. More promising models involve the interaction of the fountain clouds with material accreted from the IGM (Fraternali \& Binney, in preparation).

\section{References}

Barbieri, C.V., Fraternali, F., Oosterloo, T., Bertin, G., Boomsma, R., \& Sancisi, R. 2005, A\&A, 439, 947

Boomsma, R., Oosterloo, T., Fraternali, F., van der Hulst, J.M., Sancisi, R., 2005, ASPC, 331, 247

Bregman, J.N., 1980, ApJ, 236, 577

Fraternali, F. \& Binney, J.J., 2006, MNRAS, 366, 449

Fraternali, F., Oosterloo, T., Sancisi, R., \& Van Moorsel, G. 2001, ApJ, 562, L47

Fraternali, F., Van Moorsel, G., Sancisi, R., \& Oosterloo T. 2002, AJ, 123, 3124

Fraternali, F., Oosterloo, T., Sancisi, R., \& Swaters, R.A. 2005, ASPC, 331, 239

Heald, G.H., Rand, R.J., Benjamin, R.A., Bershady M.A. 2006, ApJ, 647, 1018

Hoopes, C.G., Walterbos, R.A.M., Rand, R.J. 1999, ApJ, 522, 669

Matthews L.D., Wood K., 2003, ApJ, 593, 721

Oosterloo T., Fraternali F., Sancisi R. 2006, AJ, nearly submitted

Rossa, J.; Dettmar, R.-J. 2003, A\&A, 406, 505

Shapiro P.R., Field G.B., 1976, ApJ, 205, 762

Strickland, D.K., Heckman, T.M., Colbert, E.J.M., Hoopes, C.G., Weaver, K.A. 2004, ApJS, 151, 193

Swaters, R.A., Sancisi, R., \& van der Hulst J. M. 1997, ApJ, 491, 140

Wakker B.P, van Woerden H., 1997, ARA\&A, 35, 217

Westmeier, T., Braun, R., \& Thilker, D. 2005, A\&A, 436, 101 